\title{
Determination of radio spectra from catalogues and identification of gigahertz peaked sources using the Virtual Observatory
}

Bernd Vollmer ${ }^{1}$, Sebastian Derriere ${ }^{1}$ Thomas P. Krichbaum Thomas Boch $^{1}$, Brice Gassmann ${ }^{1}$, Emmanuel Davoust ${ }^{3}$, Pascal Dubois $^{1}$, Françoise Genova ${ }^{1}$, François Ochsenbein ${ }^{1}$ and Wim van Driel $^{4}$

${ }^{1}$ CDS, Observatoire astronomique de Strasbourg, UMR 7550, 11 rue de l'Université, F-67000 Strasbourg, France, email: bvollmer@astro.u-strasbg.fr

${ }^{2}$ Max-Planck-Institut fr̈ Radioastronomie, Auf dem Hügel 69, D-53121 Bonn, Germany

${ }^{3}$ UMR 5572, Observatoire Midi-Pyrénées, 14 avenue E. Belin, F-31400 Toulouse, France

${ }^{4}$ Observatoire de Paris, Section de Meudon, GEPI, CNRS UMR 8111 and Université de Paris, 5 place Jules Janssen, F-92195 Meudon, France

We have used the 20 largest radio continuum catalogues contained in VizieR (CDS) to determine radio continuum spectra between wavelengths of $2 \mathrm{~cm}$ and $1 \mathrm{~m}$. For 67,000 out of the 3.5 million catalogued sources we could extract radio spectra with measurements at at least three independent frequencies (Vollmer et al. 2005, A\&A, 431, 1177). These have been validated by comparison with existing spectral indices from the literature. This work allowed us to investigated the compatibility between the 20 radio continuum catalogues (Vollmer et al. 2005, A\&A, 436, 757). Our radio spectra data base was searched for Gigahertz peaked source candidates, which we then observed quasi-simultaneously with the Effelsberg $100-\mathrm{m}$ radio telescope at $6 \mathrm{~cm}(4.85 \mathrm{GHz}), 2.8 \mathrm{~cm}(10.45 \mathrm{GHz})$, and $9 \mathrm{~mm}(32 \mathrm{GHz})$. This represents an efficient procedure to discover new Gigahertz peaked sources, which are believed to be AGNs at the beginning of their radio evolution. In our sample of more than 200 sources we find more than $50 \%$ bona fide GPS sources. In addition, we can estimate the percentage of variable sources in our multi-epoch sample of radio sources which show an inverted spectrum. We are generalizing the method by using VO capabilities to: $(i)$ identify pertinent radio catalogues in the VO registry using Uniform Content Descriptions (UCDs); all catalogues containing a user defined set of UCDs (e.g., PHOT_FLUX_RADIO* for a radio flux, POS_EQ_RA and POS_EQ_DEC for the position) are located in the VO registry and listed for further queries; ( $i$ i) extract relevant data, the user can easily assign a row of a given catalogue to a row of a previously defined output catalogue; and ( iii) normalize these for the determination of radio spectra; units can be converted, aconymes can be created, flags can be created, etc. This procedure allows to homogenize the information retrieved from a heterogenuous set of catalogues. For this purpose software allowing semi-automated information retrieval is being developed at the CDS within the framework of the European VO-TECH project. The potential usage of all available radio catalogues will strongly increase the number of independent radio source cross-correlations and radio spectra. Our aim is to include more than 100 radio catalogues into the radio spectra determination software. The results are progressively being made available to the community through the CDS services. 